\title{
Influence of Reconstruction Parameters During Filtered Backprojection and Ordered-Subset Expectation Maximization in the Measurement of the Left-Ventricular Volumes and Function During Gated SPECT
}

\author{
Diana D. Duarte*1-3, Marina S. Monteiro*1-3, Fatima El Hakmaoui ${ }^{2}$, John O. Prior ${ }^{2}$, Lina Vieira ${ }^{3}$, and José A. Pires-Jorge ${ }^{1}$ \\ ${ }^{1}$ Haute École de Santé Vaud-FilièreTRM, University of Applied Sciences Western Switzerland, Lausanne, Switzerland; ${ }^{2}$ Nuclear \\ Medicine Department, Centre Hospitalier Universitaire Vaudois and University of Lausanne, Lausanne, Switzerland; and ${ }^{3}$ Área \\ Cientifica de Medicina Nuclear, Escola Superior de Tecnologia da Saúde de Lisboa, Instituto Politécnico de Lisboa, Lisbon, Portugal
}

A crucial method for investigating patients with coronary artery disease (CAD) is the calculation of the left ventricular ejection fraction (LVEF). It is, consequently, imperative to precisely estimate the value of LVEF - a process that can be done with myocardial perfusion scintigraphy. Therefore, the present study aimed to establish and compare the estimation performance of the quantitative parameters of the reconstruction methods filtered backprojection (FBP) and ordered-subset expectation maximization (OSEM). Methods: A beating-heart phantom with known values of end-diastolic volume, end-systolic volume, and LVEF was used. Quantitative gated SPECT/quantitative perfusion SPECT software was used to obtain these quantitative parameters in a semiautomatic mode. The Butterworth filter was used in FBP, with the cutoff frequencies between 0.2 and 0.8 cycles per pixel combined with the orders of $5,10,15$, and 20. Sixty-three reconstructions were performed using 2, 4, 6, 8, 10,12 , and 16 OSEM subsets, combined with several iterations: 2, 4, 6, 8, 10, 12, 16, 32, and 64. Results: With FBP, the values of end-diastolic, end-systolic, and the stroke volumes rise as the cutoff frequency increases, whereas the value of LVEF diminishes. This same pattern is verified with the OSEM reconstruction. However, with OSEM there is a more precise estimation of the quantitative parameters, especially with the combinations 2 iterations $\times 10$ subsets and 2 iterations $\times 12$ subsets. Conclusion: The OSEM reconstruction presents better estimations of the quantitative parameters than does FBP. This study recommends the use of 2 iterations with 10 or 12 subsets for OSEM and a cutoff frequency of 0.5 cycles per pixel with the orders 5,10 , or 15 for FBP as the best estimations for the left ventricular volumes and ejection fraction quantification in myocardial perfusion scintigraphy.

Received Jun. 28, 2011; revision accepted Nov. 29, 2011.

For correspondence or reprints contact: José A. Pires-Jorge, Professeur HES-S2, Haute École de Santé Vaud-Filière TRM, Ave. de Beaumont 21, 1011 Lausanne, Switzerland.

E-mail: jose.jorge@hesav.ch

${ }^{*}$ Contributed equally to this work.

Published online Feb. 8, 2012.

COPYRIGHT @ 2012 by the Society of Nuclear Medicine, Inc.
Key Words: FBP; gated SPECT; OSEM; left ventricular function quantification

J Nucl Med Technol 2012; 40:29-36

DOI: 10.2967/jnmt.111.094599

A

primary cause of mortality in the Western world is coronary artery disease (CAD) (1). An important parameter for the prognosis and follow-up in patients with CAD is the estimation of the left ventricular (LV) ejection fraction (LVEF) (1). Many techniques can estimate this parameter and thus predict CAD. Nevertheless, because of its accessibility and low cost, the most common method used is echocardiography $(1,2)$. However, echocardiography may not achieve a satisfactory estimation of LVEF, especially in cases of left main or triple-vessel CAD, which is not detectable by this technique $(1,2)$, or echocardiography may not be feasible because of patient morphology (poor window). An overestimation of the LVEF value in patients with an LVEF greater than $70 \%$ and an underestimation in patients with a LVEF less than $70 \%$ has been also reported, suggesting that echocardiography may be less reliable (3). On the other hand, nuclear cardiology techniques such as gated SPECT have been proved to be accurate and highly reproducible for the measurement of LV volumes and LVEF, thus making it a reliable technique (1). Gated SPECT provides additional information such as the myocardial mass and temporal evaluation of the wall thickness $(4,5)$ and allows the calculation of end-diastolic volume (EDV) and end-systolic volume (ESV) and, through these, the measurement of LVEF (5-9).

Gated SPECT permits, in fact, the determination of global and regional function of the left ventricle $\left(4,6,10_{-}\right.$ 12); however, the measurement of LV quantitative param- 
eters is dependent on endocardial surface detection by the reconstruction algorithms $(4,5,9,11,13)$.

The reconstruction of gated SPECT data is mostly made by algorithms of filtered backprojection (FBP) (14-18). This method is fast, linear, and robust and produces reliable results (19). However, the use of FBP can result in poor image quality, with high noise and low contrast $(15,17)$. Because of these disadvantages, the methods of iterative reconstruction are frequently introduced as feasible alternatives to the reconstruction by $\operatorname{FBP}(17,19,20)$. Within these iterative methods, ordered-subset expectation maximization (OSEM) presents superior image quality and a higher signal-to-noise ratio $(13-15,20,21)$.

Nonetheless, both of these reconstruction methods are affected by their own reconstruction parameters, such as the cutoff frequency and order in FBP and the number of iterations (I) and subsets (S) in OSEM $(15,20,22-24)$. Hence, the use of different reconstruction parameters in each algorithm can influence the quantification of physiologic indices such as LVEF (20).

In this respect, the literature is limited, and there is no accordance in the selection of reconstruction parameters for the quantification of cardiac volumes and thus for LVEF. Furthermore, the existing European and American guidelines do not provide precise recommendations on choosing these parameters. Although the European Association of Nuclear Medicine states that for FBP "cutoff frequencies as per the manufacturer's recommendations, for example, 0.5 cycles/ $\mathrm{cm}$ (order 5 or 10 ) and 0.75 cycles $/ \mathrm{cm}$, respectively, can be chosen" (25) and for OSEM "... the rule of thumb of about...2 iterations for OSEM can be applied..." (26), the American Society of Nuclear Cardiology and the American College of Radiology and the Society of Nuclear Medicine simply do not provide information on this matter.

The aim of the present study was, therefore, to establish optimized values for the quantification of the cardiac volumes and LVEF by changing the reconstruction parameters of each algorithm. For this purpose, a beating-heart phantom with known volumes was used. In addition, a comparison of the estimation performance of the LV function quantitative parameters of both FBP and OSEM reconstruction methods was made. Finally, we also tried to see if we could make a recommendation based on our study of the best protocol to use for measuring LV function.

\section{MATERIALS AND METHODS}

\section{Phantom}

A dynamic heart phantom (BS Industrieelektronik \& Medizintechnik), as illustrated in Figure 1, was used in this study. The phantom comprises a torso anthropomorphic phantom with a pump and control unit. This phantom has a doublechamber membrane that represents the interior heart and the heart wall, allowing a simulation of the left ventricle. The compression and decompression of the interior membrane, simulating the cardiac cycle, is ensured by the pump and control unit, which also generates a trigger signal according to the diastolic or systolic end-phase. As specified by the manufacturer, this beating-heart phantom produces an ESV of $33.5 \mathrm{~mL}$, an EDV of $108.5 \mathrm{~mL}$, and an LVEF of $69 \%$ (27).

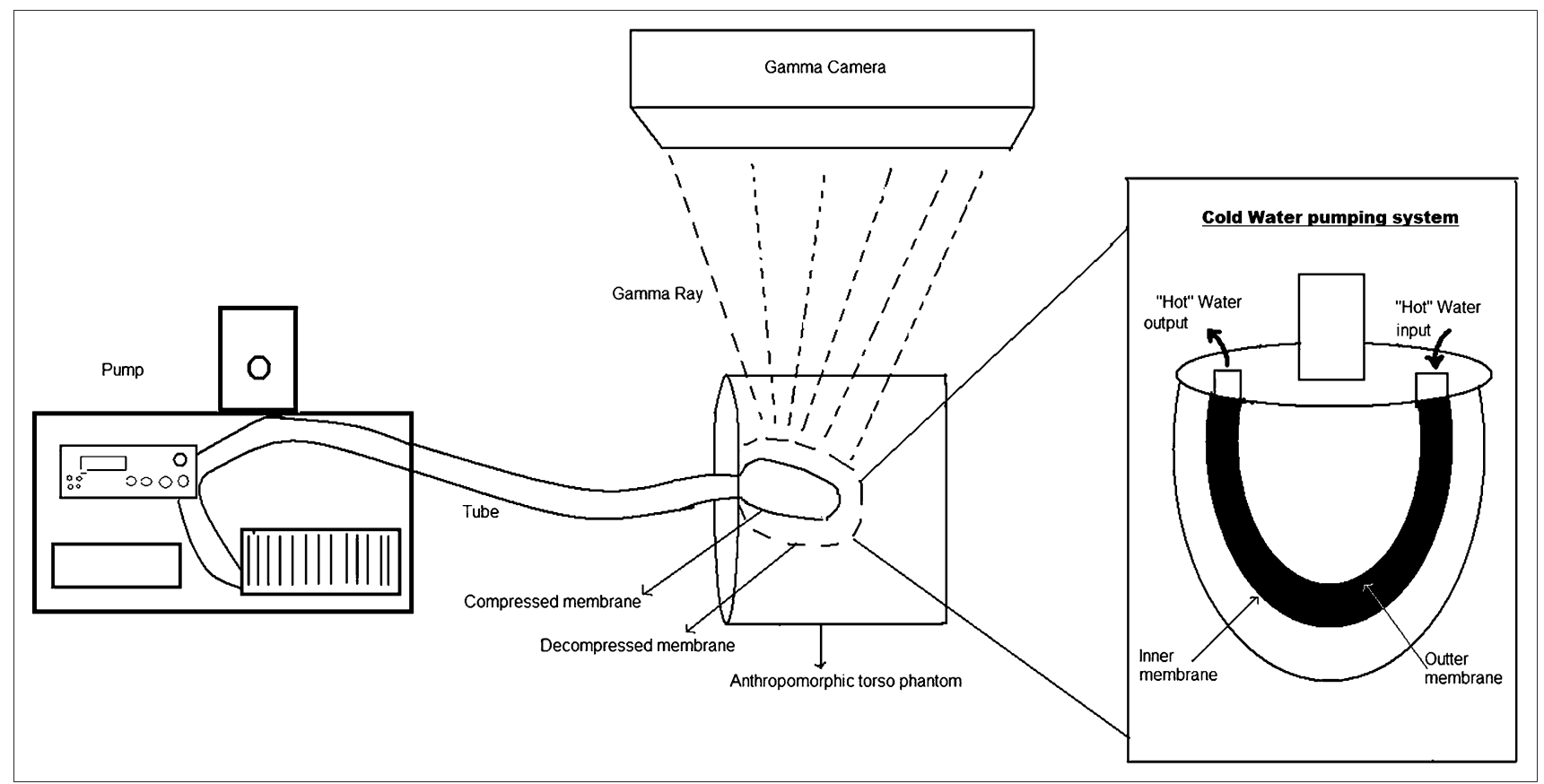

FIGURE 1. Scheme of beating-heart phantom: LV of heart is simulated by double-elastic membrane forming inner cavity filled with distilled water and outer cavity filled with air. Interior compartment represents ventricular cavity, and inside of double membrane represents cardiac wall and is filled with radioactive solution. Pumping unit guarantees periodic expansion of interior compartment, allowing volume changes of this cavity simulating cardiac cycle. 


\section{Image Acquisition}

Gated SPECT images were acquired on an E-CAM dualhead $\gamma$-camera (Siemens Medical Systems Inc.) with a lowenergy high-resolution collimator at the Nuclear Medicine Department of the Centre Hospitalier Universitaire Vaudois at Lausanne, Switzerland. The phantom was filled with $30 \mathrm{MBq}$ of ${ }^{99 \mathrm{~m}} \mathrm{Tc}$ and positioned so that the heart was centered within the field of view; this activity was chosen to represent the estimated activity from the heart in a healthy patient from the first injection in a 1-d study. The image acquisition parameters were the same as the ones used in clinical application, with a rotation angle of $90.0^{\circ}, 64 \times 64$ matrix, and zoom of 1.45 (pixel size, $6.59 \mathrm{~mm}$ ). The acquisition arc consisted of 64 projections of $40 \mathrm{~s} /$ projection. A single $20 \%$ energy window was used at $140 \mathrm{keV}$. The electrocardiographic gated SPECT image was acquired with 8 frames per cardiac cycle with a heartbeat frequency of 60 beats per minute and an end-systolic trigger signal.

\section{Gated SPECT Analysis}

To reduce interoperator variability, images were processed by only 1 user. A Xeleris (GE Healthcare) workstation was used with quantitative gated SPECT (QGS) software in a semiautomatic mode (version 2007-0.0.6.5.9; Cedars-Sinai Medical Center). Prefiltering was performed by FBP with a Butterworth filter of the 10th order, with a cutoff frequency of 0.5 cycles per pixel. After reorientation of the reconstructed data, the QGS analysis was applied to automatically calculate the volumes and LVEF.

Afterward, all the data were reconstructed with the parameters selected for both FBP and OSEM. No attenuation correction was applied.

\section{FBP Reconstruction}

FBP reconstructions were performed with a Butterworth filter. Four different orders were selected $(5,10,15$, and 20) and combined with 7 cutoff frequencies of $0.2,0.3,0.4,0.5$, $0.6,0.7$, and 0.8 cycles per pixel. For each cutoff frequency and order, the quantitative parameters (EDV, ESV, and LVEF) and images were registered. The stroke volume (SV) value was manually calculated.

\section{OSEM Reconstruction}

Sixty-three OSEM reconstructions were performed with 2, $4,6,8,10,12$, and 16 subsets. For each subset, 2, 4, 6, 8, 10, $12,16,32$, and 64 iterations were chosen. In every expectation maximization (EM) equivalent iteration, defined as the product of iterations multiplied by subsets $(I \times S)$, the EDV, ESV, and LVEF values were registered. After the analysis of these results, it was decided that another reconstruction with OSEM using the EM equivalent iteration values of $12,14,16$, $18,20,22,24,26,28,30$, and 32 would be performed. Once more, all quantitative parameters were registered.

The SV was, as with the FBP reconstruction, calculated manually.

\section{Contrast}

A contrast evaluation was performed during OSEM reconstructions for the following combinations: $8 \mathrm{I} \times 2 \mathrm{~S}$,
$4 \mathrm{I} \times 4 \mathrm{~S}, 4 \mathrm{I} \times 6 \mathrm{~S}, 2 \mathrm{I} \times 10 \mathrm{~S}$, and $2 \mathrm{I} \times 12 \mathrm{~S}$. Frame 10 was chosen from the same midventricular end-diastolic shortaxis slice to draw 5 regions of interest (ROIs) with $2 \times 2$ pixels. The background ROI was drawn at the center of the cavity, whereas the 4 remaining ROIs were drawn at the septal, inferior, lateral, and anterior walls. Contrast was defined as (signal - background)/background, where the signal is the average count of the 4 myocardial ROIs and the background is the average count of the background ROI.

\section{Statistical Analysis}

The values presented are expressed in absolute ejection fraction units and in milliliters for the volumes. These results are quoted as mean $\pm \mathrm{SD}$.

All statistical analyses were performed using the Stata 9.2 statistical data program (StataCorp LP). The significance of the difference between 2 groups of data was assessed by a nonparametric Spearman test. A $P$ value of 0.05 or less was considered significant.

To understand the difference between the values measured and the true values given by the phantom, the relative error $(\%)$ was calculated as the difference between the measured and true value divided by the true value.

\section{RESULTS}

FBP

Table 1 shows the range of volume and LVEF when reconstruction was performed with FBP obtained with the 4 orders used in each frequency $(5,10,15$, and 20). The mean presented is the mean of the quantitative values of the orders, and the $\rho$-value is the Spearman coefficient that expresses the correlation between the orders of the cutoff frequency and each quantitative parameter. The values of EDV, ESV, and SV tend to augment with higher cutoff frequencies, although they are always lower than the volumes given by the phantom. These values stabilize with a cutoff frequency of 0.6 cycles per pixel. On the other hand, the value of the LVEF tends to diminish as the cutoff frequency increases, also stabilizing with a cutoff frequency of 0.6 cycles per pixel. However, all values are higher than the LVEF of the beating-heart phantom (69\%).

Figure 2 reports the influence of the order, as the cutoff frequency increases, on the difference between the measured value and the true value, given by the relative error (\%). The figure shows that the major error is seen with a cutoff frequency of 0.2 cycles per pixel whereas the trends in the curves are the same with a cutoff frequency of 0.6 cycles per pixel.

\section{OSEM}

Table 2 indicates the range of volume and LVEF when reconstruction was performed with OSEM using the 9 different iterations and the various numbers of subsets. The calculated mean is the mean of the quantitative values of the number of iterations, and the $\rho$-value is the Spearman coefficient that expresses the correlation between the numbers of iterations and the quantitative parameters in each subset number. As seen with FBP reconstruction, also with 


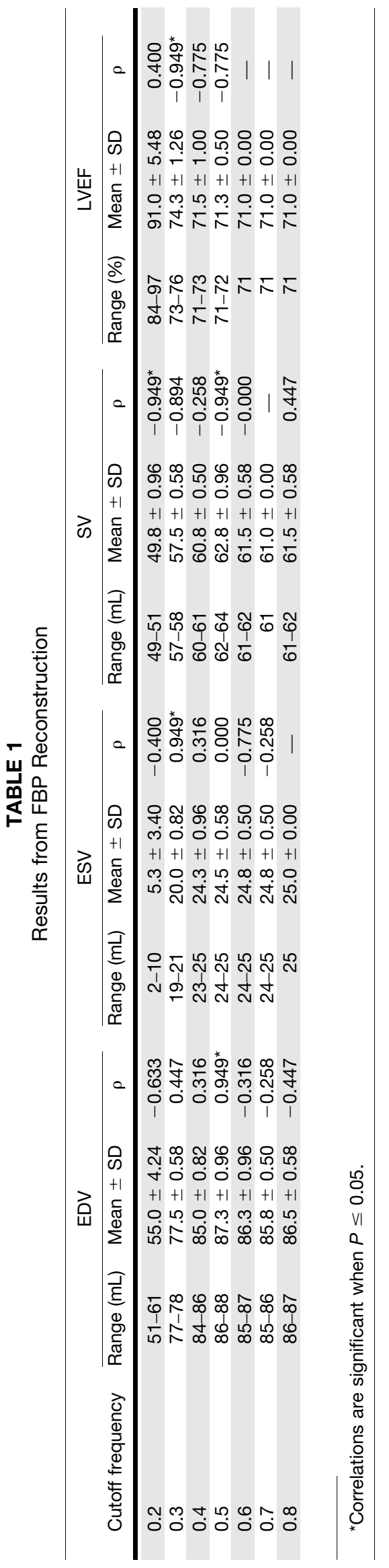

OSEM the volumes increase with an increase in the number of subsets. This means that the LVEF decreases as the number of subsets increases. Nevertheless, the LVEF values obtained with OSEM reconstruction are much closer to the true value than the values acquired with FBP. In fact, there were 6 different combinations of subsets and iterations that provided an LVEF value of exactly $69 \%$, the value considered the true value. These combinations were $8 \mathrm{I} \times 2 \mathrm{~S}, 4 \mathrm{I} \times$ $4 \mathrm{~S}, 4 \mathrm{I} \times 6 \mathrm{~S}, 2 \mathrm{I} \times 8 \mathrm{~S}, 2 \mathrm{I} \times 10 \mathrm{~S}$, and $2 \mathrm{I} \times 12 \mathrm{~S}$, resulting in 16 , $16,24,16,20$, and $24 \mathrm{EM}$ equivalent iterations, respectively.

Figure 3 demonstrates the relative error in LVEF measured with OSEM as the number of iterations and subsets varies and shows a trend to stabilization of all subsets after 12 iterations and a slight underestimation of the LVEF in most combinations. The main error seen is when 2 subsets are combined with fewer than 6 iterations.

\section{EM Equivalent Iterations}

Because of the results obtained in a previous study, we decided to perform a small study about EM equivalent iterations. Studying EM equivalent iterations from 12 to 32 in intervals of 2 iterations, as seen in Table 3, we saw a small variation between the volumes and LVEFs as the number of EM equivalent iterations increased. In addition, as shown in Figure 4, the relative error in LVEF is small.

\section{DISCUSSION}

Ejection fraction, a parameter that is accurately and reliably estimated using myocardial perfusion imaging (12), has become extremely important for reaching a fundamental diagnosis and predicting prognosis, especially of CAD $(5,28-30)$.

The most common technique to measure ejection fraction is echocardiography because it is easily accessed and inexpensive. However, this technique is not easily reproducible; calculation of its volumes is operatordependent, and after myocardial infarction the LV shape can be changed, making it difficult to achieve a good estimation of cardiac volumes (1).

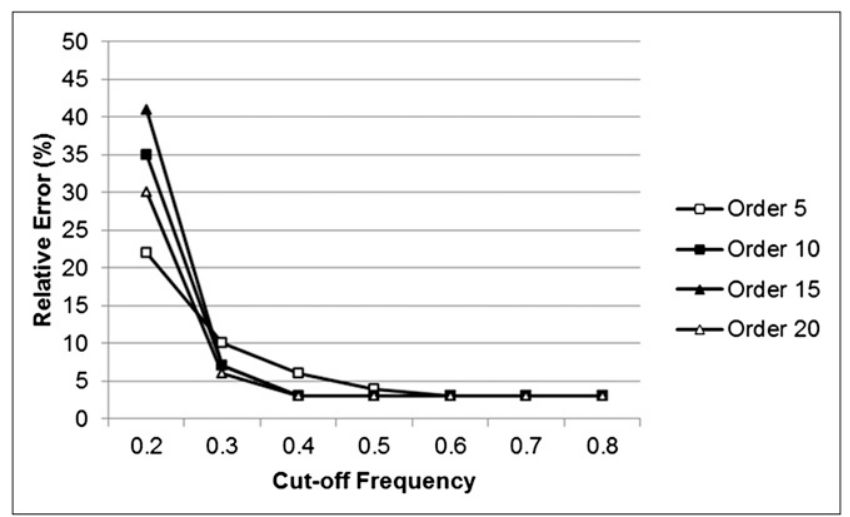

FIGURE 2. Effect of order on difference from true value of LVEF and value measured (\%) and cutoff frequency in FBP reconstruction. 
In contrast, myocardial gated SPECT is an accurate, operator-independent, repeatable, and reproducible technique for the assessment of global LV function $(1,12)$. Besides, it allows a simultaneous acquisition of quantitative and function perfusion in the same image $(1,12)$.

To achieve these results with gated SPECT, an optimal reconstruction technique is needed. The traditional reconstruction method, FBP, is still the most frequently used reconstruction method because of its speed and the fact that it is relatively computationally nonintensive $(26,31,32)$.

However, although reconstruction with OSEM is not widespread in nuclear cardiology, application of this method has been growing because it reduces noise and may include correction of physical effects such as attenuation and scatter (14,24-26).

The parameters of both these reconstruction methods can modify the quantitative results of myocardial perfusion imaging (e.g., the LVEF) $(4,7,10,11,29)$.

The Butterworth filter has been established as the best filter for nuclear cardiology applications $(7,25,31)$ because it offers good parametric variability for defining sharp edges and smoothing (33). However, regarding its parameters, the European (25) and American guidelines (26) are not explicit and there is no accordance between authors concerning the best cutoff frequency or order for the Butterworth application in nuclear cardiology. For example, Sankaran et al. (34) determined that the best cutoff frequency for nuclear cardiology was 0.32 cycles/cm, whereas Wright et al. (11) reported a cutoff frequency of 0.14 cycles $/ \mathrm{cm}$ using an eighth-order Butterworth filter.

Also, for OSEM, there is no agreement in the parameters used. Ceriani et al. (35) reported 8 subsets and 10 iterations as performing best in nuclear cardiology, whereas Seret (20) considered 8 subsets and 2 iterations the best. In addition, the American guidelines do not mention OSEM parameters, and the information in the European guidelines is limited.

For determination of a phantom's volumes and LVEF after reconstruction, QGS software has been shown to be fast (8) and reproducible $(10,12)$ and gives LVEF values that compare well with those obtained by other reliable

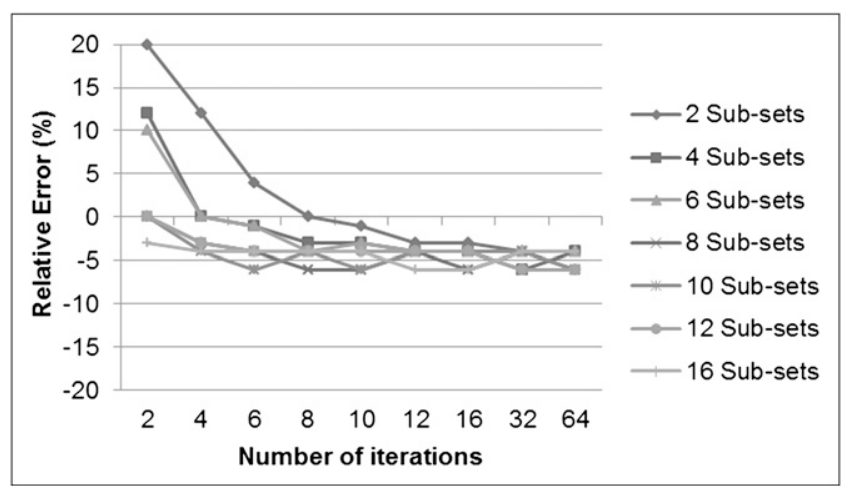

FIGURE 3. Effect of number of subsets on difference from true value of LVEF and value measured (error) and number of iterations in OSEM reconstruction. 
TABLE 3

Results from EM Equivalent Iterations

\begin{tabular}{lcccc}
\hline $\begin{array}{c}\text { EM equivalent } \\
\text { iterations }(I \times S)\end{array}$ & EDV $(\mathrm{mL})$ & ESV $(\mathrm{mL})$ & SV $(\mathrm{mL})$ & LVEF $(\%)$ \\
\hline 12 & 78 & 23 & 55 & 71 \\
14 & 81 & 24 & 57 & 70 \\
16 & 83 & 26 & 57 & 71 \\
18 & 79 & 22 & 57 & 69 \\
20 & 83 & 26 & 57 & 69 \\
22 & 83 & 26 & 59 & 67 \\
24 & 88 & 29 & 57 & 69 \\
26 & 83 & 26 & 60 & 67 \\
28 & 90 & 30 & 58 & 67 \\
30 & 86 & 28 & 60 & 67 \\
32 & 90 & 30 & 57.64 & 68.73 \\
Mean & 84.00 & 26.36 & 1.50 & 1.56 \\
SD & 4.07 & 2.69 & $0.835^{*}$ & $-0.860^{*}$ \\
$\rho$ & $0.867^{*}$ & $0.839^{*}$ & & \\
\hline${ }^{*}$ Correlations are significant when $P \leq 0.05$. & & & \\
\hline
\end{tabular}

techniques $(1,5,6,8,10)$. Nevertheless, it has been shown that QGS underestimates LV volumes $(4,12,29,35)$, resulting in an overestimation of LVEF (29).

The results achieved in this study demonstrate larger volumes, as the cutoff frequency is increased using FBP. On the other hand, the LVEF decreases with higher frequencies. These results have been confirmed by other authors, who reached the same conclusions while studying QGS performance with FBP reconstruction $(10,33,35)$.

With the lowest frequency studied, 0.2 cycles per pixel, small volumes were obtained, resulting in a large value for LVEF, an average of $91 \%$. These results, with a relative error of up to $-90 \%$ for ESV and $40 \%$ for LVEF, as seen in Figure 2, are due to the vast smoothing of the image (33). This smoothing constitutes a hindrance for the definition of wall limits by the algorithm, causing the calculation of the volumes and consequently of the LVEF to be incorrect.

With higher frequencies, that is, higher than 0.6 cycles per pixel, the difference between the volumes and LVEF obtained with these 3 different cutoff frequencies is almost nonexistent. Higher frequencies, coupled with the fact that there is a nonsignificant correlation $(\rho=-0.15, P=0.43)$ between the LVEF and the orders as seen in Table 1, reveals the minor influence of the surrounding data point on a matrix point in the final reconstruction (33).

Analyzing the influence of order number on LVEF calculation in Figure 3, we found that the highest influence occurs at lower frequencies, for which there is a large discrepancy mainly in the lowest frequency studied. This finding is explained by the fact that the order allows for optimization and control of the smoothness-sharpness proportion of the image (33), showing a higher influence on the smoother images that are obtained with a low cutoff frequency. Therefore, the curves show a trend toward a decrease in the relative error, lowering it below 5\%, and are the same if a cutoff frequency of 0.6 cycles per pixel is used. Although this is a good approximation of the true value given by the phantom, the quality of the quantitative information given by these higher frequencies is unreliable because there is a suppression of information (33).

Because of these findings, it is generally agreed that the best cutoff frequency to study LV quantitative parameters with FBP reconstruction would be 0.5 cycles per pixel (24). As for the orders, the results of this study suggest that orders of 10,15 , or 20 be used because there is no major difference in their influence on the cutoff frequency of 0.5 cycles per pixel or above.

Overall, with the FBP reconstruction, LVEFs are overestimated and volumes underestimated. The overestimation of LVEF is close to $3 \%$ above the true value (using a cutoff frequency of 0.5 cycles/pixel).

As for OSEM reconstruction, Table 2 verifies that with an increase in the number of subsets and iterations, all volumes tend to diminish whereas LVEF values increase.

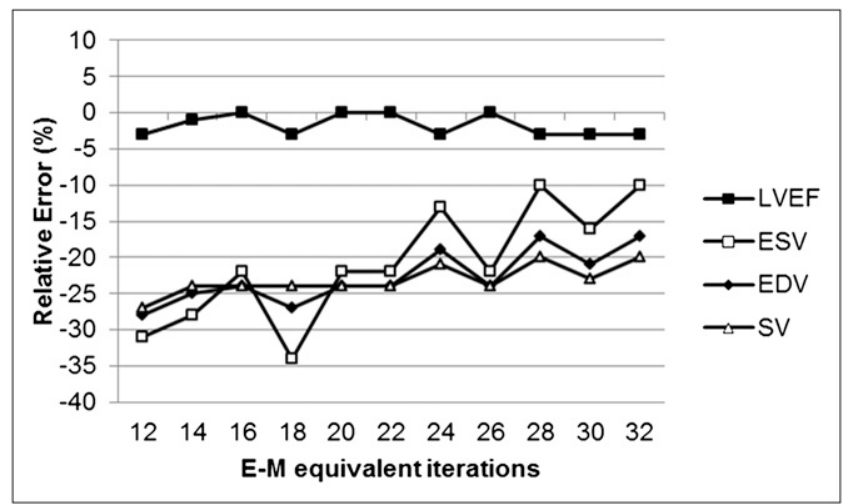

FIGURE 4. Effect of number of EM equivalent iterations on difference from true value of volumes and ejection fraction of LV and value measured (error) in OSEM reconstruction. 
When establishing a correlation between the numbers of subsets and LVEF, we saw a moderate negative correlation ( $\rho=-0.45, P=0.0002 ; 95 \%$ confidence interval, -0.63 to -0.23 ), whereas between the numbers of iterations and LVEF, there was a substantial negative correlation $(\rho=$ $-0.64 ; P=0.0001 ; 95 \%$ confidence interval, -0.76 to -0.46 ). Because these correlation values were within the confidence interval, this might mean that the LVEF was more influenced by the number of subsets than by the number of iterations. Moreover, the lower the number of subsets is, the higher is the rate of influence of the number of iterations, which is reflected by a strongly significant correlation. This significant correlation means that a small change in the number of iterations within a low subset value can result in a large disparity within the obtained volumes and LVEF.

As can be seen in Figure 3, with higher numbers of subsets the volumes and LVEF tend to stabilize into values under a relative error of 5\%. In fact, the reconstruction with the OSEM algorithm shows values with a small relative error $(<5 \%)$, with the exception of 4 or 6 subsets, with several iterations lower than 4 , and 2 subsets, with an iteration number lower than 8 .

For the best estimation of LVEF by OSEM, as also seen in Figure 3, there are 6 different combinations of iterations and subsets that lead to an LVEF of $69 \%$. These combinations result in values of EM equivalent iterations that are close, expressly 16, 20, and 24.

To enhance and guarantee that these results were, in fact, the best possible estimation, we performed another study to analyze the variation of the volumes and LVEF according to a small range of EM equivalent iterations. This range, in agreement with the EM equivalent iterations previously obtained for an LVEF closer to the true value, varied from 12 to $32 \mathrm{EM}$ equivalent iterations.

Within this range, as seen in Table 3, the maximum variation is only $1.56 \%$ on the LVEF value. The maximum relative error obtained was $3 \%$, considerably lower than the results obtained with other combinations for OSEM.

As confirmed by Figure 4, there is a permanent underestimation of the volumes with these numbers of EM equivalent iterations. As a small increase of the EM equivalent iterations occurs, for example, from 20 to $32 \mathrm{EM}$ equivalent iterations, there is a significant augmentation of all the volume values and a decrease in the LVEF values.

Figure 4 shows that the ESV values obtained with 18, 24, and $32 \mathrm{EM}$ equivalent iterations oscillate from the normal trend to an increase as the number of iterations augments. The results were tested twice with another reconstruction made by the same operator to confirm the findings, and identical values were obtained. These findings might be explained by the inability of the reconstruction algorithm to establish the contour limits of the small $(33.5 \mathrm{~mL})$ systolic volume because of poor resolution (27).

Although the results achieved with the numbers of EM equivalent iterations are good for an estimation of quantitative parameters, qualitative parameters should also be considered when dealing with patients with heart defects. For this reason, as well as to restrict a smaller interval of EM equivalent iterations, it was decided to measure image contrast. The images, chosen on the basis of the best estimation for the quantitative parameters, had different combinations of subsets and iterations-specifically $8 \mathrm{I} \times$ $2 \mathrm{~S}, 4 \mathrm{I} \times 4 \mathrm{~S}, 4 \mathrm{I} \times 6 \mathrm{~S}, 2 \mathrm{I} \times 10 \mathrm{~S}$, and $2 \mathrm{I} \times 12 \mathrm{~S}$ - to study image contrast with low iterations and with a low number of subsets.

Contrast values were 5.57 for $2 \mathrm{I} \times 10 \mathrm{~S}$ and $2 \mathrm{I} \times 12 \mathrm{~S}, 5.50$ for $4 \mathrm{I} \times 4 \mathrm{~S}$ and $4 \mathrm{I} \times 6 \mathrm{~S}$, and 5.45 for $8 \mathrm{I} \times 2 \mathrm{~S}$. Although the difference between these values is small, as expected because these are already the best estimations achieved for the calculation of LVEF and associated volumes, the best contrast result would be obtained with larger subsets values and lower iteration numbers.

On the basis of these findings and the observations in Table 3 and Figure 4, the best iteration or subset combination for the OSEM method to estimate the quantitative parameters of myocardial perfusion SPECT was $2 \mathrm{I} \times 10 \mathrm{~S}$, although $2 \mathrm{I} \times 12 \mathrm{~S}$ also had good results. In fact, this last combination has been proved the best combination for OSEM that gives the most consistent results with angiography, according to Bitarafan and Rajabi (5).

Finally, when FBP reconstruction was compared with the OSEM method in this study, we noted that although FBP overestimates the LVEF value, OSEM estimates it precisely, with just a slight underestimation.

In addition, taking into account the noise reduction and better image quality given by the OSEM reconstruction in nuclear cardiology, this study suggests the use of this iterative method to reconstruct images from myocardial perfusion scintigraphy.

As a limitation of this study, the reconstructions made with OSEM and FBP were performed with only 1 phantom acquisition and not with real patients. Nevertheless, because a phantom with exactly known values was used and the QGS software presents high reproducibility, this limitation was acceptable.

As a future approach-a possible complement to this study - a dedicated study to confirm the suggested parameters obtained in our results for OSEM with other standard techniques, such as the MRI, in patients with CAD should be performed. In addition, a study with a model with regional wall motion abnormalities using a region-independent phantom is recommended, employing the method described in this study. Also, for validation of OSEM as the recommended reconstruction method for gated SPECT in nuclear cardiology, a comparative study between different processing software is proposed.

\section{CONCLUSION}

Myocardial gated SPECT provides precise diagnostic and prognostic information for patients with CAD. It is a technique that is accurate and reproducible for the measurement of cardiac volume and LVEF. As demonstrated with the present study, myocardial gated SPECT 
allows a precise LVEF estimation if the optimal reconstruction parameters are used.

Although the FBP method is faster and still is the most frequently used in daily practice, the best results in this study were achieved with the iterative reconstruction method. In general, OSEM provides a slight underestimation of the LVEF true value. However, this estimation is more precise than the overestimation seen with FBP. In our attempt to select the combinations of $2 \mathrm{I} \times 10 \mathrm{~S}$ or $2 \mathrm{I} \times 12 \mathrm{~S}$, our findings suggest several EM equivalent iterations within 12-32 for OSEM.

Thus, we recommend the use of the iterative reconstruction method with these parameters, when available, even if FBP was initially set up for historical reasons. For nuclear medicine departments in which OSEM would not be available, we would advise the use of the FBP reconstruction method with the Butterworth filter, with a cutoff frequency of 0.5 cycles per pixel and an order of 10,15, and 20.

\section{ACKNOWLEDGMENTS}

We acknowledge Fabrice Camus and Gilles Allenbach for their help with the phantom acquisition and Vincent Dunet for his time and effort in assisting with the statistical data. We are also very grateful to the full team of the Department of Nuclear Medicine at CHUV for their support, encouragement, and availability during the development of this study

\section{REFERENCES}

1. De Winter O, Sutter JD, Dierckx RA. Clinical relevance of left ventricular volume assessment by gated myocardial SPET in patients with coronary artery disease. Eur J Nucl Med Mol Imaging. 2002;29:957-966.

2. Fleming RM. High-dose dipyridamole and gated sestamibi SPECT imaging provide diagnostic resting and stress ejection fractions useful for predicting the extent of coronary artery disease. Angiology. 2002;53:415-421.

3. Fleming RMA. Tate-en-Tate comparison of ejection fraction and regional wall motion abnormalities as measured by echocardiography and gated sestamibi SPECT. Angiology. 2002;53:313-321.

4. Visser JJN, Sokole EB, Verberne HJ, et al. A realistic 3-D gated cardiac phantom for quality control of gated myocardial perfusion SPET: the Amsterdam gated (AGATE) cardiac phantom. Eur J Nucl Med Mol Imaging. 2004;31:222-228.

5. Bitarafan A, Rajabi H. The effect of filtrating and reconstruction method on the left ventricular ejection fraction derived from GSPET: a statistical comparison of angiography and echocardiography. Ann Nucl Med. 2008;22:707-713.

6. Yalçin H, Maza S, Yalçin F. Single photon emission computed tomography: an alternative imaging modality in left ventricular evaluation. Vasc Health Risk Manag. 2008;4:1069-1072.

7. Vakhtangandze T, Hall DO, Zananiri MR, Rees MR. The effect of Butterworth and Metz reconstruction filters on volume and ejection fraction calculations with 99Tcm gated myocardial SPECT. Br J Radiol. 2005;78:733-736.

8. He X, Links J, Frey E. An investigation of the trade-off between the count level and image quality in myocardial perfusion SPECT using simulated images: the effects of statistical noise and object variability on defect detectability. Phys Med Biol. 2010;55:4949-4961.

9. Van der Veen BJ, Scholte A, Schneider P, Stokkel M. The consequences of a new software package for the quantification of gated-SPECT myocardial perfusion studies. Eur J Nucl Med Mol Imaging. 2010;37:1736-1744.

10. Hambye AS, Vervaet A, Dobbeleir A. Variability of the left ventricular ejection fraction and volumes with quantitative gated SPECT: influence of algorithm, pixel size and reconstruction parameters in small and normal-sized hearts. Eur J Nucl Med Mol Imaging. 2004;31:1606-1613.

11. Wright GA, McDade M, Martin W, Hutton I. Quantitative gated SPECT: the effect of reconstruction filter on calculated left ventricular ejection fractions and volumes. Phys Med Biol. 2002;47:N99-105.
12. Fredericks N, Baxter P, McKay E, Smart R. An assessment of the sensitivity of the Cedars-Sinai quantitative Gated SPECT software to changes in the reconstruction of the short-axis slices. J Nucl Med Technol. 1999;27:123-126.

13. Zakavi S, Zonnoozi A, Kakhki V, Hajizadeh M, Momennezhad M, Ariana K. Image reconstruction using filtered backprojection and iterative method: effect on motion artifacts in myocardial perfusion SPECT. J Nucl Med Technol. 2006;34:220-223.

14. Seret A, Forthomme J. Comparison of different types of commercial filtered backprojection and ordered-subset expectation maximization SPECT reconstruction software. J Nucl Med Technol. 2009;37:179-187.

15. Brambilla M, Cannillo B, Dominietro M, Leva L, Secco C, Inglzse E. Characterization of ordered-subsets expectation maximization with $3 \mathrm{D}$ post-reconstruction Gauss filtering and comparison with filtered backprojection in ${ }^{99 \mathrm{~m}} \mathrm{Tc}$ SPECT. Ann Nucl Med. 2005;19:75-82.

16. Yusoff MNS, Zakaria A. Relationship between the optimum cut off frequency for Butterworth filter and lung-heart ratio in ${ }^{99} \mathrm{~m} T \mathrm{c}$ myocardial SPECT. Iran J Radiat Res. 2010;8:17-24.

17. Miller T, Sampathkumaran K. Digital filtering in nuclear medicine. J Nucl Med. 1982;23:66-72.

18. Søndergaard HM, Madsen MM, Boisen K, et al. Evaluation of iterative reconstruction (OSEM) versus filtered back: projection for the assessment of myocardial glucose uptake and myocardial perfusion using dynamic PET. Eur J Nucl Med Mol Imaging. 2007;34:320-329.

19. Vanhove C, Defrise M, Franken PR, Everaert H, Deconinck F, Bossuyt A. Interest of the ordered subsets expectation maximization (OS-EM) algorithm in pinhole single-photon emission tomography reconstruction: a phantom study. Eur J Nucl Med. 2000;27:140-146.

20. Seret A. The number of subsets required for OSEM reconstruction in nuclear cardiology. Eur J Nucl Med Mol Imaging. 2006;33:231.

21. Reilhac A, Tomeï S, Buvat I, Michel C, Keheren F, Costes N. Simulation-based evaluation of OSEM iterative reconstruction methods in dynamic brain PET studies. Neuroimage. 2008;39:359-368.

22. Norberg P, Bake B, Jacobsson L, Carlsson GA, Gustafsson A. Evaluation of reconstruction techniques for lung single photon emission tomography: A Monte Carlo study. Nucl Med Commun. 2007;28:929-936.

23. Gilland DR, Tsui BMW, McCartney WH, Perry JR, Berg J. Determination of the optimum filter function for SPECT imaging. J Nucl Med. 1988;29:643-650.

24. Hutton BF, Hudson HM, Beekman FJ. A clinical perspective of accelerated statistical reconstruction. Eur J Nucl Med. 1997;24:797-808.

25. Broek W, Cuocolo A, Ghilardi A, et al. Myocardial Perfusion Imaging: A Technologist's Guide. Vienna, Austria: European Association of Nuclear Medicine; 2004.

26. Hesse B, Tägil K, Cuocolo A, et al. EANM/ESC procedural guidelines for myocardial perfusion imaging in nuclear cardiology. Eur J Nucl Med Mol Imaging. 2005;32:855-897.

27. BS Industrieelektronik \& Medizintechnik. Dynamic Heart Phantom. July 2009. Available at: http://www.bsi-schoeppy.de/download/pdf/Herzphantom_engl_07_ 2009.pdf. Accessed January 20, 2012.

28. Germano G, Kiat H, Kavanagh PB, et al. Automatic quantification of ejection fraction from gated myocardial perfusion SPECT. J Nucl Med. 1995;36: 2138-2147.

29. Lavender FM, Meades RT, Al-Nahhas A, Nijran KS. Factors affecting the measurement of left ventricular ejection fraction in myocardial perfusion imaging. Nucl Med Commun. 2009;30:350-355.

30. Nakajima K, Higuchi T, Taki J, Kawano M, Tonami N. Accuracy of ventricular volume and ejection fraction measured by gated myocardial SPECT: comparison of 4 software programs. J Nucl Med. 2001;42:1571-1578.

31. Yusoff MNS, Zakaria A. Determination of the optimum filter for qualitative and quantitative ${ }^{99 \mathrm{~m}}$ Tc myocardial SPECT imaging. Iran J Radiat Res. 2009;6: $173-182$.

32. Hansen CL, Goldstein RA, Akinboboye OO, et al. Myocardial perfusion and function: single photon emission computed tomography. J Nucl Cardiol. 2007; 14:e39-60.

33. Van Laere K, Koole M, Dierckx R. Image filtering in single-photon emission computed tomography: principles and applications. Comput Med Imaging Graph. 2001;25:127-133.

34. Sankaran S, Frey EC, Gilland KL, Tsui BMW. Optimum compensation method and filter cutoff frequency in myocardial SPECT: a human observer study. $\mathrm{J} \mathrm{Nucl}$ Med. 2002;43:432-438.

35. Ceriani L, Ruberto T, Delaloye AB, Prior JO, Giovanella L. Three-dimensional ordered-subset expectation maximization iterative protocol for evaluation of left ventricular volumes and function of left ventricular volumes and function by quantitative gated SPECT: a dynamic phantom study. J Nucl Med Technol. 2010; $38: 18-23$. 